\title{
Genetic Analysis and Mapping of QTLs for Soybean Biological Nitrogen Fixation Traits Under Varied Field Conditions
}

\section{OPEN ACCESS}

Edited by:

Hon-Ming Lam,

The Chinese University of Hong Kong,

China

Reviewed by:

Brett James Ferguson,

The University of Queensland,

Australia

Oswaldo Valdes-Lopez,

National Autonomous University

of Mexico, Mexico

Yinglong Chen,

University of Western Australia,

Australia

*Correspondence:

Hong Liao

hliao@fafu.edu.cn;

hliao@scau.edu.cn

${ }^{t}$ These authors have contributed equally to this work

Specialty section:

This article was submitted to

Plant Microbe Interactions,

a section of the journal

Frontiers in Plant Science

Received: 16 August 2018

Accepted: 17 January 2019

Published: 01 February 2019

Citation:

Yang $Q$, Yang $Y, X u R, L v H$ and

Liao H (2019) Genetic Analysis and Mapping of QTLs for Soybean Biological Nitrogen Fixation Traits

Under Varied Field Conditions.

Front. Plant Sci. 10:75

doi: 10.3389/fpls.2019.00075

\author{
Qing Yang ${ }^{\dagger}$, Yongqing Yang ${ }^{\dagger}$, Ruineng Xu, Huiyong Lv and Hong Liao* \\ Root Biology Center, College of Resources and Environment, Fujian Agriculture and Forestry University, Fuzhou, China
}

Soybean is an important economic and green manure crop that is widely used in intercropping and rotation systems due to its high biological nitrogen fixation (BNF) capacity and the resulting reduction in $\mathrm{N}$ fertilization. However, the genetic mechanisms underlying soybean BNF are largely unknown. Here, two soybean parent genotypes contrasting in BNF traits and $168 \mathrm{~F}_{9: 11}$ recombinant inbred lines (RILs) were evaluated under four conditions in the field. The parent FC1 always produced more big nodules, yet fewer nodules in total than the parent FC2 in the field. Furthermore, nodulation in FC1 was more responsive to environmental changes than that in FC2. Broad-sense heritability $\left(h^{2} b\right)$ for all BNF traits varied from 0.48 to 0.87 , which suggests that variation in the observed BNF traits was primarily determined by genotype. Moreover, two new QTLs for BNF traits, $q B N F-16$ and $q B N F-17$, were identified in this study. The qBNF-16 locus was detected under all of the four tested conditions, where it explained 15.9$59.0 \%$ of phenotypic variation with LOD values of 6.31-32.5. Meanwhile qBNF-17 explained $12.6-18.6 \%$ of observed variation with LOD values of 4.93-7.51. Genotype group analysis indicated that the FC1 genotype of qBNF-16 primarily affected nodule size (NS), while the FC2 genotype of $q B N F-16$ promoted nodule number (NN). On the other hand, the FC1 genotype of qBNF-17 influenced NN and the FC2 genotype of qBNF-17 impacted NS. The results on the whole suggest that these two QTLs might be valuable markers for breeding elite soybean varieties with high BNF capacities.

Keywords: QTLs (quantitative trait loci), BNF capacities, RILs (recombinant inbred lines), nodule number and nodule dry weight, nodule size, field conditions tests

\section{INTRODUCTION}

As an important economic crop, soybean is a main source of edible oil and protein for human around the world due to the high oil (20-25\%) and high protein (42-45\%) contents in the seeds (Aziz et al., 2016). At the same time, the high capacity of biological nitrogen fixation (BNF) found in leguminous crops, including soybean, makes this a key source of green manure in agro-ecosystems (Kumudini et al., 2008; Chen and Liao, 2017; Yang et al., 2017).

Nitrogen $(\mathrm{N})$ is one of the most limiting factors in crop production. In order to obtain high yields, farmers tend to supply excessive amounts of $\mathrm{N}$ fertilizers, which not only increases input costs, but also causes potentially adverse effects on the environment, including air and water 
pollution (Santos et al., 2013; Li et al., 2016). Although $\mathrm{N}$ is abundant in the atmosphere, plants are unable to acquire it directly on their own, because it predominantly exists in the inert form of $\mathrm{N}_{2}$. BNF is a process in which plant unavailable atmospheric $\mathrm{N}_{2}$ is converted into readily available ammonia $\left(\mathrm{NH}_{3}\right)$ in nodules formed through symbiotic associations between plants and microbes (Fox et al., 2016; Yang et al., 2017).

Chemical synthesis provides 118 million metric tons of fertilizer $\mathrm{N}$ each year (Joseph et al., 2016). In comparison, it is estimated that symbiosis between nitrogen-fixing rhizobia and plants provides 50-70 million tons of $\mathrm{N}$ for agricultural systems each year (Herridge et al., 2008). As one of the most important legume crops, soybean fixes 16.4 million tons of $\mathrm{N}$ annually, which represents $77 \%$ of the total $\mathrm{N}$ fixed by legume crops (Herridge et al., 2008). In modern agricultural systems, soybean is typically considered to have the most potential for sustaining green agricultural systems. For example, in Brazil, over $70 \%$ of the $\mathrm{N}$ required for soybean growth is derived primarily from BNF (Peoples et al., 2009). Therefore, breeding elite soybean cultivars with high BNF capacities and high yields could be an efficient way to maintain agriculture sustainability.

Soybean BNF capacity is influenced by many environmental factors. Among them, rhizobial strains play critically roles in the nitrogen fixation capacity of soybean nodules (Denison and Kiers, 2004), and which could be classed into three genotype/phenotypes: mutualistic rhizobia, parasitic rhizobia and non-symbiotic. To facilitate agriculture sustainability, mutualistic rhizobia are preferred to become parasitic and nonsymbiotic rhizobia, because that parasitic and non-symbiotic rhizobia fix little nitrogen or even unable to infect legumes at all (Denison and Kiers, 2004). For example, Yang et al. (2017) found that upon inoculation with effective rhizobial strains, nodule numbers and dry weights of a RIL population did not significantly vary compared to inoculation with less effective strains, yet shoot dry weight increased by $18.25 \%$ on average. Other research has revealed that inoculation with effective rhizobium strains in soybean not only enhances nodule fresh weight, but also increases $\mathrm{N}$ and $\mathrm{P}$ contents, as well as, yield in the field (Qin et al., 2012). These results imply that rhizobial inoculation increases soybean BNF capacity mainly due to the effects of particular rhizobial species and strains. Beyond rhizobial species, BNF capacity has also been associated with soil characteristics, such as high soil nitrate levels, which tend to reduce nitrogen fixation capacity (Nohara et al., 2005; Yang et al., 2009), and low available phosphorus, which not only limits legume growth, but also inhibits BNF capacity (Adelson et al., 2000; Ward, 2011). Furthermore, water stress also appears to affect BNF capacity (Nascimento et al., 2016; Jemo et al., 2017). In short, BNF is a complex process that is affected by host and symbiotic genotypes, along with the context of environmental conditions in which the symbiosis occurs.

High BNF efficiency mainly depends on the phenotypes expressed in symbiosis between host plants and rhizobia (Nicolás et al., 2006), including the readily observable traits of nodule number (NN), nodule weight (NW), and nodule size (NS). In a relatively stable field environment, the heritability of nodulation traits may exceed 0.8 (Yang et al., 2017), suggesting that nodulation traits are mainly controlled by genetic loci. To date, a number of QTLs for nodulation traits have been identified at different stages of soybean development in pots or in field. These QTLs are distributed on several linkage groups (LGs), including D1b, A1, C2, O, B1, H, B2, E, J, and I (Tanya et al., 2005; Nicolás et al., 2006; Santos et al., 2013; Yang et al., 2017). Some QTLs, such as $q B N F-C 2, q B N F-O$ and $q B N F-B 1$, are co-located with yield trait loci (Yang et al., 2017), implying that BNF capacity might affect soybean yield. Therefore, breeding new soybean varieties that produce higher yields through optimization of BNF capacity promises to be a feasible and economic strategy. Unfortunately, possibly due to technical limitations and labor costs, most of the QTLs for BNF traits obtained from field observations are preliminary and minor, so they are not likely to be useful as markers in soybean breeding programs. As a result, progress in studying BNF associated QTL markers in field experiments has lagged behind marker studies for other soybean traits.

Despite the volume of research devoted to mining QTLs for soybean BNF capacity in the field, fine mapping of QTLs regulating nodulation traits in soybean still remain largely unknown. In the present study, two soybean genotypes contrasting in nodulation traits and $168 \mathrm{~F}_{9: 11}$ recombinant inbred lines (RILs) bred from their progeny were grown in the field to evaluate the effects of $\mathrm{N}$ level and rhizobial inoculation on nodulation and BNF traits, as well as, to identify associated QTLs.

\section{MATERIALS AND METHODS}

\section{Plant Materials and Field Trials}

In this study, a soybean population consisting of $168 \mathrm{~F}_{9: 11}$ RILs was derived from a cross between two accessions contrasting in BNF capacity, FC1 (a cultivar accession with high BNF capacity) and FC2 (a semi-wild landrace with low BNF capacity), using the single seed descent (SSD) method. This population was further used to construct a genetic linkage map to detect QTLs associated with BNF traits, as well as, to evaluate effects of $\mathrm{N}$ level and rhizobial inoculation on BNF traits. A field trial under four environmental conditions was carried out at the Zhaoxian experimental farm $\left(\mathrm{E} 114.48^{\circ}, \mathrm{N} 37.50^{\circ}\right)$ of the Institute of Genetics and Developmental Biology, Chinese Academy of Sciences. The four environmental conditions included with $(+\mathrm{R})$ or without rhizobial inoculation (-R) at two field sites contrasting in long-term nitrogen fertilization ( $\mathrm{HN}$ : high $\mathrm{N}$; LN: low N). Three highly effective rhizobium strains, BXYD3, BXBL9, and BDYD1, which were previously identified belonging to Bradyrhizobium elkanii based on morphological and $16 \mathrm{~s}$ ribosomal DNA sequence analysis (Cheng et al., 2009), were used as mixture inoculants in this study. The rhizobial inoculation treatment was applied to seeds as described by Qin et al. (2012). Briefly, soybean seeds were uniformly mixed with rhizobium inoculants before planting. The soil at the experiment site is a Fluventic Ustochrept soil. Basic characteristics of the top $20 \mathrm{~cm}$ of soil in the HN and LN field are shown in Table 1. The previous crop was wheat, which was fertilized with $202.5 \mathrm{~kg} \cdot \mathrm{ha}^{-1}$ of $\left(\mathrm{NH}_{4}\right)_{2} \mathrm{HPO}_{4}$ and $187.5 \mathrm{~kg} \cdot \mathrm{ha}{ }^{-1}$ of urea as base fertilizer, and $75 \mathrm{~kg} \cdot \mathrm{ha}^{-1}$ of urea as additional fertilizer during the elongation 
TABLE 1| Basic characteristics of the field soils where the tested soybean lines were grown.

\begin{tabular}{|c|c|c|c|c|c|}
\hline Field & $\mathrm{pH}$ & Nitrate $\mathbf{N}\left(\mathbf{m g} \cdot \mathbf{k g}^{-1}\right)$ & Available P $\left(\mathbf{m g} \cdot \mathbf{k g}^{-1}\right)$ & Available K $\left(\mathbf{m g} \cdot \mathbf{k g}^{-1}\right)$ & Organic matter $\left(\mathbf{g} \cdot \mathbf{k g}^{-1}\right)$ \\
\hline $\mathrm{HN}$ & $8.12 \pm 0.10$ & $90.25 \pm 9.51$ & $16.17 \pm 2.96$ & $89.53 \pm 12.53$ & $1.93 \pm 0.20$ \\
\hline LN & $8.04 \pm 0.08$ & $58.17 \pm 7.10$ & $14.70 \pm 2.24$ & $87.75 \pm 8.50$ & $1.97 \pm 0.27$ \\
\hline
\end{tabular}

stage in the HN field. No fertilizer was applied during soybean growth. Field management (i.e., pest control and irrigation, etc.) followed local farmer practices. Parental genotypes and RILs were planted in a split plot design with plots arranged in randomized complete blocks within each block of split plots. There were two plots for each RILs in each treatment, and in total, were 1,360 plots in the field. Thirty seeds were sown per plot in three $3 \mathrm{~m}$ rows spaced $0.5 \mathrm{~m}$ apart.

\section{Plant Sampling and Measurements}

At the R4 stage, three representative plants from each plot were harvested, and in total, six plants were harvested for each line in each treatment. Plant roots were manually extracted from the soil to ensure the integrity of the root systems. All nodules were removed and cleared carefully from roots prior to drying in a $37^{\circ} \mathrm{C}$ oven. Three nodulation traits, namely NN, NW, and NS, were investigated for subsequent analysis. NS was calculated by the quotient of total NW and NN. NN and NW were derived from individual plant. The plants were dried at $60^{\circ} \mathrm{C}$, and then measured dry weight and total $\mathrm{N}$ content (TNC) using a continuous flow analyzer as described by Bao (2000). The TNC was used to represent the BNF capacity.

\section{Genotyping by High-Throughput Sequencing}

Genomic DNA of the two parents were extracted using commercial extraction kit, DNeasy Plant Mini Kit (QIAGEN, Germany), following the manufacturer protocol. Library construction and sequencing were performed on the Illumina sequencing platform. Briefly, genomic DNA was fragmented to 100-300 bp size and two replicate genomic DNA re-sequencing libraries (100-300 bp insert) were prepared for two entire lanes sequencing on Hiseq X10 PE150 sequencing system. Genotyping by sequencing (GBS) libraries were constructed using the EcoRI and NIaIII enzymes modified from the Elshire's protocol (Elshire et al., 2011). The simple description as follows: after genomic DNA extracting, $100 \mathrm{ng}$ of DNA for each plant were used for digesting reaction with EcorI and NIaIII (New England Biolabs, Ipswich, MA, United States) in 96-well plates. Then, the reactions were mixed with about $25 \mathrm{pmol}$ of unique A1 and A2 adapters per well. The libraries in 96-well plates were then pooled, further DNA fragments size 400-600 bp were isolated on a $1 \%$ agarose gel and purified using a PCR purification kit (NEB), and then amplified for 12 cycles using Phusion DNA polymerase (NEB). Finally, the pooled libraries were adjusted to $10 \mathrm{nmol}$ and sequenced with PE125 on the HiSeq4000 (Illumina, San Diego, CA, United States). Both genomic DNA re-sequencing and GBS were performed by Genedenovo Biotechnology Co., Ltd. (Guangzhou, China).

\section{SNP Identification and Bin Map Construction}

For SNPs calling, the Burrows-Wheeler Aligner (BWA) was used to align the clean reads from each plant against the reference genome Glycine max Wm82.a2.v1 with the settings 'mem 4 -k 32 -M' (Li and Durbin, 2009). Genotypes of all samples were determined by using the GATK's Unified Genotyper. SNPs were filtered using GATK's Variant Filtration with follow parameters (-Window 4, -filter "QD $<2.0\|F S>60.0\| \mathrm{MQ}<40.0$," -G_filter "GQ $<20$ ") and SNPs with segregation distortion or sequencing errors were eliminated. The physical positions of each SNP were further determined by using the software tool ANNOVAR (Wang et al., 2010). Polymorphic parental markers were recorded as male genotype "a" and female genotype "b," other segregation patterns were recorded as missing data "-." However, the reading depth of SNP variants outside the range of 5-1,500 would be also considered as missing data. After that, Chi-square $\left(\chi^{2}\right)$ tests were conducted for all SNPs to detect segregation distortion. The sliding window $(1 \mathrm{~kb})$ approach was used to evaluate groups of consecutive SNPs for utility in genotyping and avoidance of false positive population based SNPs. Qualified bins of SNP markers were then used to construct genetic linkage maps using Join Map 4.1. The regression algorithm and Kosambi mapping function were used in marker distance calculation. The LOD value was set according to the chromosome numbers. A Perl SVG module was used to draw the linkage map. Bin markers were named as Ch" $\mathrm{x}$ ". " $y$ " according to results of SNP calling, " $\mathrm{x}$ " and "y" represent "chromosome number" and "physical position," respectively.

\section{QTL Detection}

Multiple-QTL model (MQM) implemented in MapQTL6.0 (Van Ooijen, 2009) was used to detect QTLs for nodulation and BNF capacity traits. The critical logarithm of odds (LOD) threshold was set to 4.5 for declaring the significance of a QTL in a particular genomic region. A total of 1,000 permutations with $P$-values $<0.05$ was used to verify LOD scores. QTLs for which the LOD score simultaneously exceeded 4.5 and the genome wide LOD, were considered as high confidence QTLs in MQM mapping.

\section{Statistical Analysis}

Data for the three BNF traits were used for variance and QTL analysis. Analysis of variance (ANOVA) was implemented in the QTL ICIMapping V4.1 software (Meng et al., 2015). Parental genotypes were also planted with six replications, which were analyzed separately from the RILs. Broad sense heritability $\left(h^{2}{ }_{b}\right)$ was estimated for each trait according to: $h^{2}{ }_{b}=\mathrm{VG} /(\mathrm{VG}+\mathrm{VE})$, with VG as the variance between RILs and VE as the variance 
within RILs. The Student's $t$-test was used to test the significance of the effects of $\mathrm{N}$ level and rhizobial inoculation on each trait in RILs. Multi-factor and multivariate analysis of variance (ANOVA) was also performed using SPSS 19 (Colin and Paul, 2012).

\section{RESULTS}

\section{Phenotype Evaluation of Two Parents}

The two parental genotypes, FC1 and FC2, contrasted in root growth and nodulation in the field under natural conditions. ANOVA revealed significant genotypic variation between parental genotypes for NN $(P<0.001)$ and NS $(P<0.01)$, as well as, a significant interaction between rhizobium inoculation and genotype on NW $(P<0.05)$ (Table 2). Compared with FC2, FC1 had a shallower root architecture denoted by more roots in the top soil, and more big nodules than FC2 in the field (Figures 1A-C). However, FC2 formed more nodules than FC1 in each environment, as indicated by $83.90-$ $1473.09 \%$ differences in NN under the four tested environments (Figure 1D). Interestingly, NW of FC2 was only higher than that of FC1 in plots without rhizobial inoculation (-R), but not in $\mathrm{HN}+\mathrm{R}$ or $\mathrm{LN}+\mathrm{R}$ (Figure 1E). Nodules on FC1 roots were much bigger than on FC2 roots in all treatments except HN-R, with NS being $450.11 \%, 260.89 \%$, and $382.50 \%$ bigger for FC1 compared to $\mathrm{FC} 2$ in $\mathrm{HN}+\mathrm{R}, \mathrm{LN}-\mathrm{R}$, and $\mathrm{LN}+\mathrm{R}$, respectively (Figure 1F), indicating that $\mathrm{FC} 1$ was more conducive to nodule development in response to rhizobial inoculation.

In addition, $\mathrm{N}$ level significantly influenced nodulation on FC1 but not FC2. NN increasing by $535.71 \%$ or $41.94 \%$, NW increasing by $323.70 \%$ or $235.96 \%$, and NS increasing by $12.90 \%$ and $181.63 \%$ in response to rhizobium inoculation in LN and $\mathrm{HN}$ treatments, respectively (Figures 1D-F). These results strongly suggest that nodulation traits are much more sensitive to environment changes for FC1 than for FC2, which might be controlled by genetic loci.

\section{Phenotypic Variation Among Recombinant Inbred Lines (RILs)}

As shown in Table 3, significant phenotypic variation existed for all three nodulation traits within the $168 \mathrm{~F}_{9: 11}$ soybean RILs under different environments. As expected, among the RILs, high $\mathrm{N}$ supply significantly suppressed nodule formation and development as indicated by declines of $33.31 \%$ and $30.91 \%$ in $\mathrm{NN}, 63.72 \%$ and $63.72 \%$ in NW, and $29.87 \%$ and $51.98 \%$ in NS, with or without rhizobial inoculation, respectively (Figures 2A-C). Furthermore, TNC increased in response to rhizobial inoculation at both $\mathrm{N}$ levels, with a greater impact in LN than in HN (Figure 2D), suggesting that BNF capacity is inhibited by high $\mathrm{N}$ supply in the RIL population.

The mean value for each trait among all RILs was between the mean values of the two parents, while the maximum and minimum values were beyond the extremes of the two parents. Distributions for the nodulation traits under all conditions were approximately normal according to Kurtosis and Skewness values calculated over six replicates. Broad-sense heritability $\left(h^{2}{ }_{b}\right)$ for all the traits under the tested environments varied from 0.48 to 0.87 , with generally higher values being observed in LN than in $\mathrm{HN}$ plots (Table 3). Overall, the results herein suggested that variations in nodulation traits mainly depended on genotype.

\section{Construction of Genetic Linkage Map}

After strict screening, 168 strains of the RIL population were accepted and genotyped using a sliding-window method (Chang and Lee, 2004). Bin markers for each chromosome were screened, and a total of 3,319 recombinant bin markers were identified (Table 4). A genetic map was constructed by mapping these 3,319 bin markers onto the 20 soybean chromosomes (Supplementary Figure S1). The complete genetic distance of the linkage map was $2,537.6 \mathrm{cM}$. The average distance between two adjacent markers was $0.76 \mathrm{cM}$. Among the 20 soybean chromosomes, chromosome 7 had the highest number of bin markers at 194, which cover a genetic length of $157.9 \mathrm{cM}$. Chromosome 10 held the fewest markers, 100, over a distance of $101.0 \mathrm{cM}$. The $W$ gene located on chromosome 13 has been identified in previous work as responsible for flower color (Takahashi et al., 2010). In order to examine the fidelity of the genetic map constructed herein, this flower color gene was mapped relative to the bin markers used in the current study. As expected, the gene encoding flower color was mapped as the $W$ gene located on chromosome 13 at bin marker Chr13.17168452, with a high LOD value of 74.56 (Supplementary Figure S1). This clearly demonstrated that the linkage map constructed in this study is a high quality map that can be used in further studies.

\section{QTL Identification for Nodulation Traits}

A total of 12 significant QTLs explaining $12.6-59 \%$ of the phenotypic variation observed among the $168 \mathrm{~F}_{9: 11}$ soybean RILs were identified for nodulation traits in the field under four environment conditions (Table 5). The LOD values of these QTL varied from 4.93 to 32.52. However, 10 of the QTLs were closely mapped on Chromosome 16, and the other two QTLs were mapped on Chromosome 17 at two very closely linked locations. These results indicating that all of the significant QTLs might be controlled by two genetic loci, which were, therefore, named as $q B N F-16$ and $q B N F-17$. Among them, $q B N F-16$ is a stable and major QTL, which was detected under all four of the environment conditions, and explained $40.9 \%-59 \%$ and $15.9 \%-$ $45.4 \%$ of the variation in NS and NN, respectively. The additive effects of $q B N F-16$ for NS and NN were derived from FC1 and FC2, respectively. In contrast, $q B N F-17$ was only detected in LN$\mathrm{R}$, and it explained $18.6 \%$ and $12.6 \%$ of the variation in $\mathrm{NN}$ and NW, respectively. The additive effects of $q B N F-17$ for NN and NW were derived from FC1 and FC2, respectively. As for interactions between traits, the results in this study indicated that $\mathrm{NN}$ is negatively correlated with NS.

\section{Effects of Genotype on Nodulation Traits Under Varied Environmental Conditions}

Biological nitrogen fixation capacity can be represented by plant total nitrogen content (TNC) (Li et al., 2016). In order to evaluate the effects of $q B N F-16$ and $q B N F-17$ on nodulation traits, the 
TABLE 2 | ANOVA for variation in biological nitrogen fixation traits between two parental genotypes.

\begin{tabular}{|c|c|c|c|c|c|c|c|}
\hline Traits & $\mathbf{R}$ & $\mathbf{N}$ & $\mathbf{G}$ & $\mathbf{R} \times \mathbf{N}$ & $\mathbf{R} \times \mathbf{G}$ & $\mathbf{N}^{*} \mathbf{G}$ & $\mathbf{R} \times \mathbf{N} \times \mathbf{G}$ \\
\hline NN & 0.54 & 0.53 & 5.03E-06* & 0.36 & 0.13 & 0.13 & 0.87 \\
\hline NW & 0.22 & 0.26 & 0.94 & 0.21 & $0.02^{*}$ & 0.12 & 0.52 \\
\hline NS & 0.17 & 0.88 & $0.01^{*}$ & 0.71 & 0.13 & 0.99 & 0.6 \\
\hline
\end{tabular}

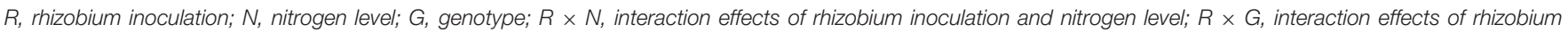

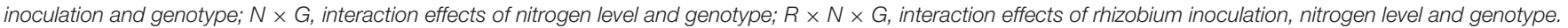
Asterisks indicated significant differences through ANOVA at the 5\% (*) level.
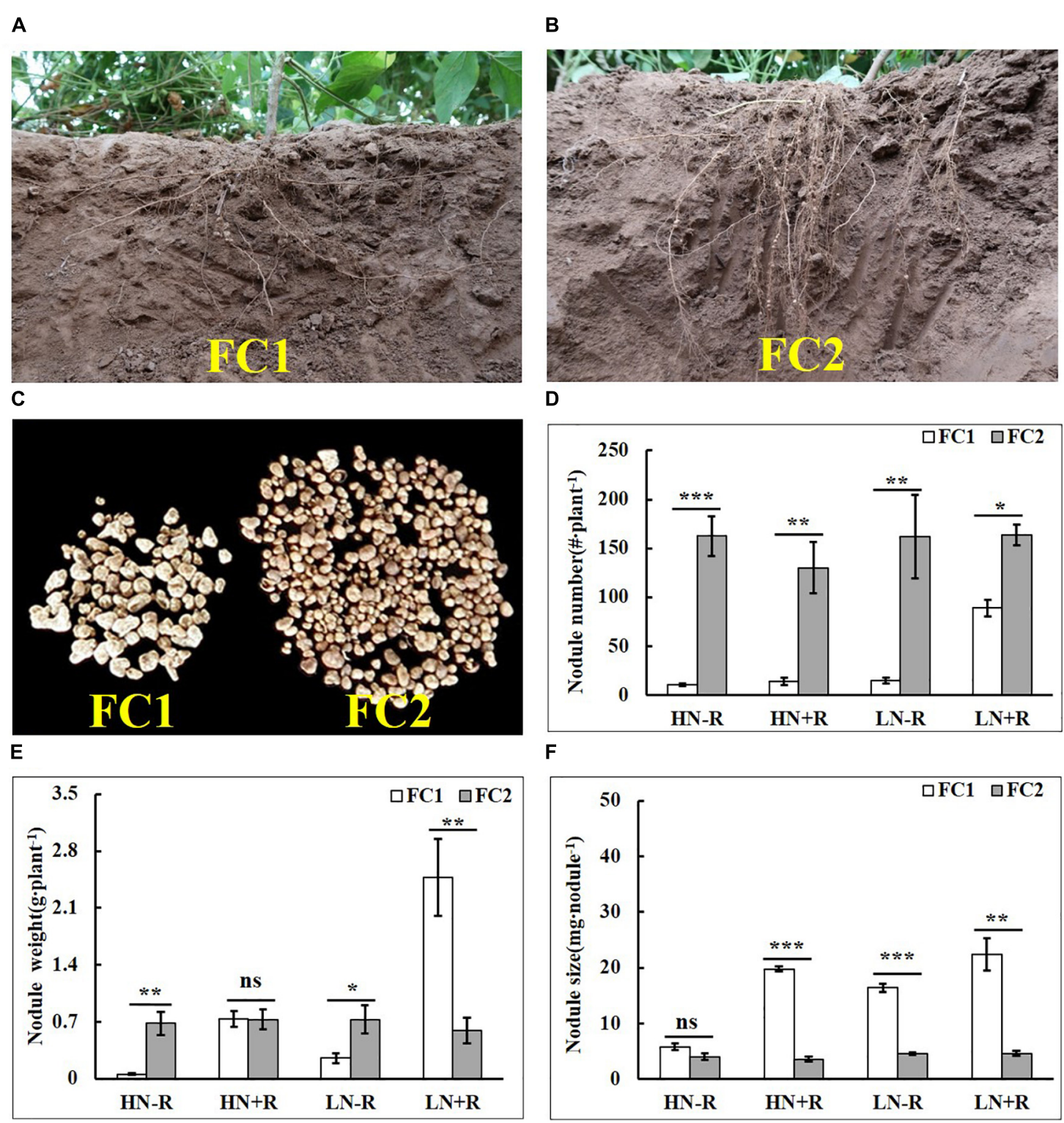

FIGURE 1 | Root and nodule characteristics for FC1 and FC2 soybean parental lines in the field under four environment conditions. (A,B) Root and nodule characteristics of FC1 and FC2. (C) Nodule size and number for FC1 and FC2 under natural conditions. (D-F) Nodule number, nodule weight, and nodule size on roots of FC1 and FC2 under four environments. Bars represented means \pm SE from six replications. Asterisks indicated significant differences between FC1 and FC2 in the Student $t$-test at $5 \%\left(^{*}\right), 1 \%\left(^{* *}\right)$ and $1 \%\left(^{* *}\right)$ levels, while "ns" represented no significant difference. "HN" and "LN" denoted high and low nitrogen, "+R" and "-R" denoted the inoculation or non-inoculation of rhizobium. 
TABLE 3 | Phenotypic variation and genetic analysis of biological nitrogen fixation traits using 168 F9:11 soybean RILs under different field conditions.

\begin{tabular}{|c|c|c|c|c|c|c|c|c|c|c|}
\hline \multirow[t]{2}{*}{ Trait } & \multirow[t]{2}{*}{ Environment } & \multicolumn{2}{|c|}{ Parents } & \multicolumn{7}{|c|}{ RILs } \\
\hline & & FC1 & FC2 & Mean \pm SD & Min & Max & CV\% & $h_{b}^{2}$ & Skew & Kurt \\
\hline \multirow[t]{4}{*}{ NN (\#·plant $\left.{ }^{-1}\right)$} & HN-R & $10.33 \pm 3.01$ & $162.5 \pm 49.49$ & $119.45 \pm 129.22$ & 1 & 985 & 108.18 & 0.76 & 0.76 & -0.05 \\
\hline & $\mathrm{HN}+\mathrm{R}$ & $14 \pm 9.64$ & $130.2 \pm 65.08$ & $131.10 \pm 116.15$ & 1 & 938 & 88.60 & 0.48 & 0.88 & 0.26 \\
\hline & LN-R & $14.67 \pm 6.66$ & $162 \pm 103.68$ & $156.36 \pm 164.38$ & 1 & 1047 & 105.13 & 0.83 & 1.02 & 0.31 \\
\hline & $\mathrm{LN}+\mathrm{R}$ & $89 \pm 21$ & $163.67 \pm 26.86$ & $174.78 \pm 130.44$ & 4 & 835 & 74.63 & 0.63 & 0.65 & -0.33 \\
\hline \multirow[t]{4}{*}{ NW (g.plant ${ }^{-1}$ ) } & $\mathrm{HN}-\mathrm{R}$ & $0.06 \pm 0.02$ & $0.68 \pm 0.35$ & $0.43 \pm 0.40$ & 0 & 2.51 & 91.96 & 0.62 & 1.01 & 1.03 \\
\hline & $\mathrm{HN}+\mathrm{R}$ & $0.74 \pm 0.24$ & $0.73 \pm 0.31$ & $0.66 \pm 0.57$ & 0 & 5.78 & 85.96 & 0.69 & 0.78 & 0.15 \\
\hline & LN-R & $0.25 \pm 0.15$ & $0.73 \pm 0.42$ & $0.72 \pm 0.50$ & 0 & 3.56 & 70.08 & 0.67 & 0.49 & 0.12 \\
\hline & $\mathrm{LN}+\mathrm{R}$ & $2.48 \pm 1.16$ & $0.6 \pm 0.39$ & $1.06 \pm 0.70$ & 0 & 4.16 & 66.54 & 0.70 & 1.12 & 1.45 \\
\hline \multirow[t]{4}{*}{ NS (mg.nodule ${ }^{-1}$ ) } & HN-R & $5.83 \pm 1.57$ & $4.01 \pm 1.4$ & $6.36 \pm 6.30$ & 0.16 & 53.8 & 99.03 & 0.70 & 1.57 & 2.99 \\
\hline & $\mathrm{HN}+\mathrm{R}$ & $36.77 \pm 29.41$ & $3.6 \pm 1.17$ & $7.12 \pm 7.13$ & 0.2 & 87.38 & 100.2 & 0.75 & 1.38 & 1.37 \\
\hline & LN-R & $16.42 \pm 1.77$ & $4.55 \pm 0.63$ & $9.93 \pm 9.77$ & 0.26 & 81.21 & 98.34 & 0.87 & 1.64 & 2.98 \\
\hline & $L N+R$ & $22.35 \pm 7.1$ & $4.63 \pm 1.19$ & $8.81 \pm 7.86$ & 0 & 73.15 & 89.19 & 0.86 & 1.36 & 1.81 \\
\hline
\end{tabular}

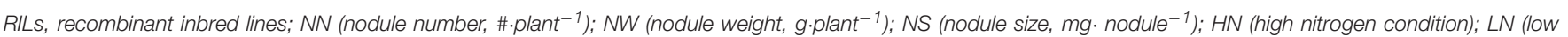

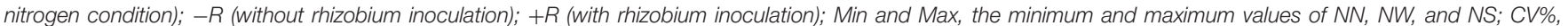
coefficient of variation; $h^{2}$, broad-sense heritability; Skew and Kurt, Skewness and Kurtosis.

A

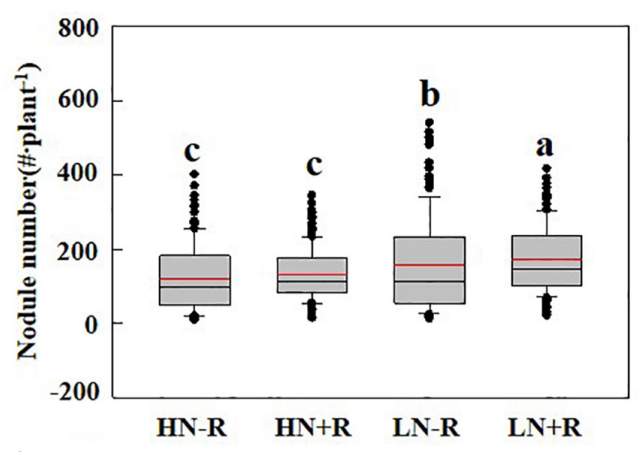

C

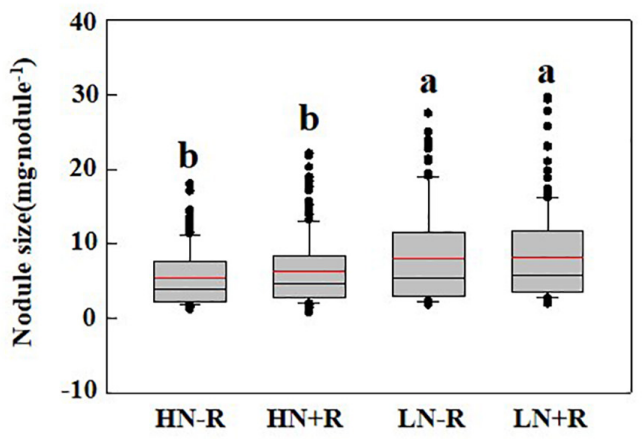

B

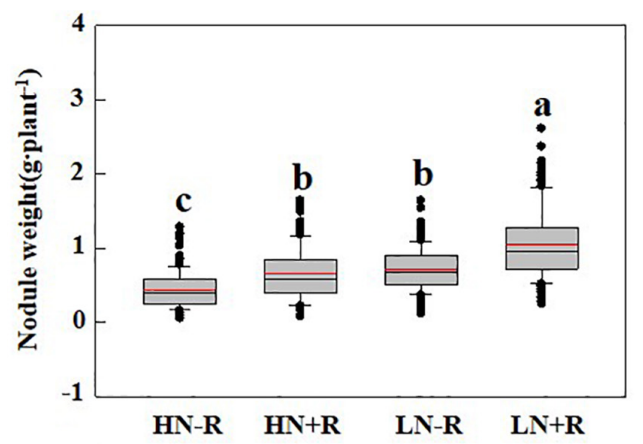

D

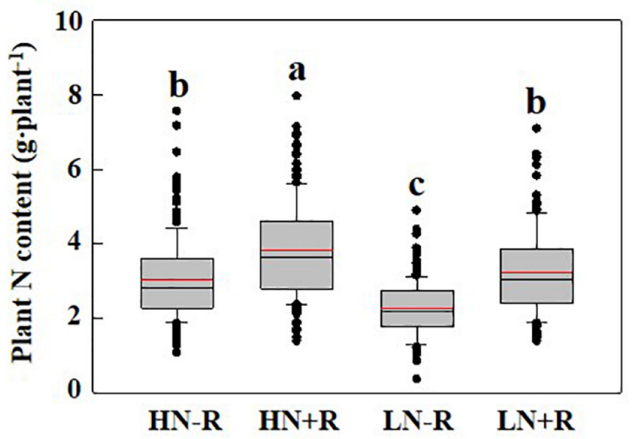

FIGURE 2 | Biological nitrogen fixation (BNF) traits as affected by N level and rhizobium in RILs. (A) Nodule number; (B) nodule weight; (C) nodule size; and (D) plant nitrogen content. The black and red lines, lower and upper edges, and bars above or below the boxes represented median and mean values, 25th, 75th, 5th, and 95th percentiles of all data, respectively. Different letter(s) over error bars indicated significance differences between HN-R, HN+R, LN-R, and LN+R conditions through the Student $t$-test at the $1 \%$ level.

$168 \mathrm{~F}_{9: 11}$ lines were classed into two distinct genotypic groups based on bin markers Chr16.37147484 and Chr17.12638388. Then, comparative analysis of nodulation traits and TNC were conducted among different environments between these two groups.
The genotype of $q B N F-16$ significantly affected NN and NS, but not NW and TNC (Figure 3). The FC1 group had fewer, yet larger nodules than the FC2 group (Figures $3 \mathrm{~A}, \mathrm{C}$ ). This suggested that the FC1 genotype of $q B N F-16$ supported nodule development, while the FC2 genotype of $q B N F-16$ promoted 
TABLE 4 | Summary of bin marker characteristics of recombinant inbred line (RIL) population.

\begin{tabular}{|c|c|c|c|c|}
\hline Chr & $\begin{array}{c}\text { Genetic } \\
\text { distance/cM }\end{array}$ & $\begin{array}{l}\text { Physical } \\
\text { length }\end{array}$ & $\begin{array}{c}\text { Number of } \\
\text { bin } \\
\text { markers }\end{array}$ & $\begin{array}{c}\text { Average } \\
\text { coverage } \\
\text { distance/cM }\end{array}$ \\
\hline Chr01 & 123.1 & 56510449 & 169 & 0.73 \\
\hline ChrO2 & 131.0 & 47706575 & 165 & 0.79 \\
\hline ChrO3 & 140.0 & 45730193 & 169 & 0.83 \\
\hline ChrO4 & 158.1 & 51405255 & 188 & 0.84 \\
\hline Chr05 & 97.5 & 41919006 & 140 & 0.70 \\
\hline Chr06 & 152.2 & 50892625 & 177 & 0.86 \\
\hline ChrO7 & 157.9 & 43534570 & 194 & 0.81 \\
\hline Chr08 & 186.5 & 47709553 & 174 & 1.07 \\
\hline Chr09 & 155.5 & 50173153 & 131 & 1.19 \\
\hline Chr10 & 101.0 & 51542279 & 100 & 1.01 \\
\hline Chr11 & 168.5 & 34671664 & 110 & 1.53 \\
\hline Chr12 & 119.4 & 40030102 & 178 & 0.67 \\
\hline Chr13 & 121.8 & 45514498 & 175 & 0.70 \\
\hline Chr14 & 83.7 & 47841279 & 171 & 0.49 \\
\hline Chr15 & 75.0 & 51710377 & 191 & 0.39 \\
\hline Chr16 & 76.1 & 37192783 & 178 & 0.43 \\
\hline Chr17 & 137.0 & 41348852 & 178 & 0.77 \\
\hline Chr18 & 103.2 & 57961359 & 182 & 0.57 \\
\hline Chr19 & 82.3 & 50522110 & 175 & 0.47 \\
\hline Chr20 & 167.7 & 47868276 & 174 & 0.96 \\
\hline Total & 2537.6 & 941784958 & 3319 & 0.76 \\
\hline
\end{tabular}

nodule formation. Furthermore, the FC1 genotype of $q B N F-16$ was responsive to rhizobial inoculation, as indicated by increases of $\mathrm{NN}$ of $93.20 \%$ and $55.34 \%$, NW of $126.97 \%$ and $87.08 \%$, and TNC of $25.18 \%$ and $56.56 \%$ upon rhizobial inoculation in $\mathrm{HN}$ and LN plots, respectively. Meanwhile, NS was only affected by $\mathrm{N}$ level. The FC2 genotype of $q B N F-16$ was also influenced by $\mathrm{N}$ level, as showed by decreases in NN of $42.04 \%$ or $24.07 \%$, and in NW of $64.69 \%$ or $51.69 \%$ in HN plots with or without rhizobial inoculation, respectively.
The genotype of $q B N F-17$ also only significantly affected NN and NS, but had opposite effects compared to $q B N F-16$ (Figure 4) impacts. The FC2 group had fewer, but bigger nodules than the FC1 group (Figures 4A,C), suggesting that the FC2 genotype of $q B N F-17$ participated in nodule development, while the FC1 genotype of $q B N F-17$ advanced nodule formation. Both $\mathrm{N}$ level and rhizobial inoculation significantly impacted NW and TNC for both FC1 and FC2 genotypes of $q B N F-17$, while only $\mathrm{N}$ level affected NS, and rhizobial inoculation only influenced NN for the FC2 genotype at the $q B N F-17$ locus in LN plots.

\section{DISCUSSION}

By 2050, agricultural production might need to be increased by $70 \%$ in order to satisfy the needs of a growing population (Joseph et al., 2016). In order to achieve higher yields, farmers tend to supply excessive amounts of fertilizers, which often leads to environmental problems (Li et al., 2016). Legumes, particularly soybean, not only could provide oil and protein for humans, but also can improve soil quality and reduce the need for $\mathrm{N}$ fertilizers. This benefit of growing legumes is due to the unique capability among this family to participate in BNF, which converts atmospheric nitrogen $\left(\mathrm{N}_{2}\right)$ into ammonia with the assistance of rhizobia in symbiotically derived organs known as nodules (Udvardi and Poole, 2013). Identification and fine mapping QTLs for BNF capacity hold the promise of facilitating the development of soybean cultivars with higher yield potentials and superior BNF capacities that will improve our ability to meet food and environment demands.

Soybean yield has been demonstrated being largely dependent on root architecture (RA) and BNF capacity (Li et al., 2016; Chen and Liao, 2017; Yang et al., 2017), and the effects of RA and BNF capacity on yield are also associated with each other. For example, RA of legume roots is not only involved in elaboration of root system through lateral root formation, but also in nodule formation with rhizobia from soils (Yendrek et al., 2010).

TABLE 5 | Putative QTLs detected for biological nitrogen fixation traits using 168 F:11 soybean RILs in the field.

\begin{tabular}{|c|c|c|c|c|c|c|c|c|}
\hline Integrated QTL ${ }^{a}$ & Environment $^{b}$ & Separate QTL & Chr & Marker or interval ${ }^{\mathrm{c}}$ & Position (cM) & LOD & $\operatorname{Add}^{d}$ & $\operatorname{PVE}(\%)^{\mathrm{e}}$ \\
\hline \multirow[t]{10}{*}{$q B N F-16$} & $\mathrm{HN}-\mathrm{R}$ & $q N N-H N-R$ & Gm16 & Chr16.37147484 & 17.63 & 22.12 & 65.49 & 45.4 \\
\hline & & $q N S-H N-R$ & Gm16 & Chr16.37147484 & 17.63 & 25.25 & -4.78 & 50.0 \\
\hline & $\mathrm{HN}+\mathrm{R}$ & $q N N-H N+R$ & Gm16 & Chr16.37559118 & 18.55 & 6.31 & 29.19 & 15.9 \\
\hline & & $q N W-H N+R$ & Gm16 & Chr16.36986426 & 16.09 & 7.60 & -0.17 & 18.8 \\
\hline & & $q N S-H N+R$ & Gm16 & Chr16.37147484 & 17.63 & 19.18 & -3.31 & 40.9 \\
\hline & LN-R & $q N N-L N-R$ & Gm16 & Chr16.37156866 & 17.87 & 15.63 & 69.79 & 28.4 \\
\hline & & $q N S-L N-R$ & Gm16 & Chr16.37147484 & 17.63 & 30.50 & -6.01 & 56.7 \\
\hline & $L N+R$ & $q N N-L N+R$ & Gm16 & Chr16.37147484 & 17.63 & 14.91 & 55.68 & 33.6 \\
\hline & & $q N W-L N+R$ & Gm16 & Chr16.36986426 & 16.09 & 10.12 & -0.25 & 24.2 \\
\hline & & $q N S-L N+R$ & Gm16 & Chr16.37147484 & 17.63 & 32.52 & -5.08 & 59.0 \\
\hline \multirow[t]{2}{*}{$q B N F-17$} & LN-R & $q N N-L N-R$ & Gm17 & Chr17.12638388 & 61.78 & 7.51 & -55.58 & 18.6 \\
\hline & & $q N W-L N-R$ & Gm17 & Chr17.12728155- Chr17.13805621 & 60.24 & 4.93 & 0.40 & 12.6 \\
\hline
\end{tabular}

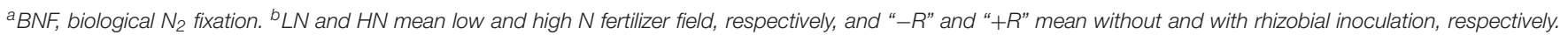

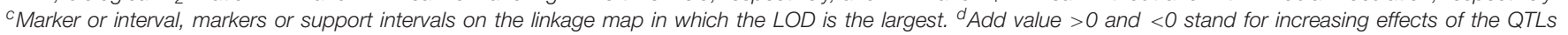
derived from FC2 and FC1, respectively. ${ }^{e}$ PVE (\%), percentage of phenotypic variance explained by the QTL. 

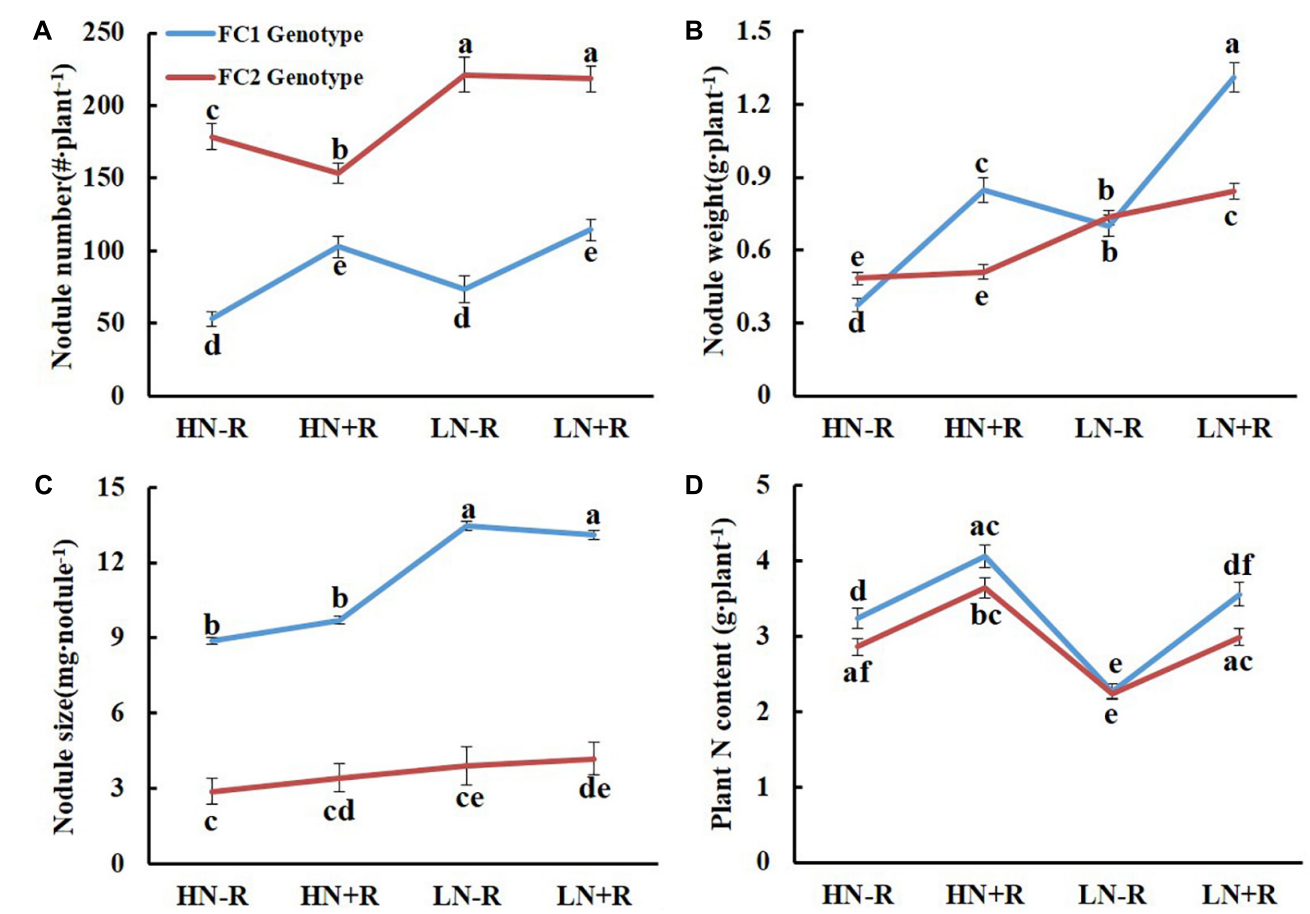

FIGURE 3 | Effects of qBNF-16 genotypes on BNF traits. (A) Nodule number; (B) nodule weight; (C) nodule size; and (D) whole plant nitrogen content. Based on SNP marker Chr16.37147484, the RIL population was divided into FC1 and FC2 genotype groups which consisted of 72 and 92 lines, respectively. The blue and red line represented mean \pm SE values for the FC1 and FC2 genotype groups. Different letter(s) over the standard error bar indicated significant differences of mean values at the $5 \%$ level.

However, information of the relationships between RA and BNF remains limited. Our recent research suggested that the soybean plants with shallow RA had more and heavier nodules, as well as higher SDW. Meanwhile, soybean roots became shallower after inoculating with rhizobia, showing strong synergistic interactions between RA and BNF (Yang et al., 2017). In this study, the two soybean accession FC1 and FC2 contrasting in RA were evaluated in varied field conditions. Interestingly, we found that the parental genotype FC1 with shallow RA preferred to form fewer but bigger nodules compared with the parental genotype FC2 with deep RA, implying that more complicate relationships between RA and BNF exited and need to be further evaluated.

Since effective rhizobial strains play critical roles in facilitating soybean production with high nitrogen fixation capacity, lots of commercial rhizobial strains have been isolated and applied in the field (Menna et al., 2006; Thuita et al., 2012; Sivparsad et al., 2015). However, none of the commercial rhizobial strains could meet the needs for all crop species and/or all cultivars in the same species in the field (Thomas-Oates et al., 2003). In this study, FC2 had a higher nodulation ability with numerous small nodules than FC1, which was possibly caused by non-selectivity compatibility of FC2 to most indigenous rhizobia, and this nonselectivity compatibility greatly related to plant species (i.e., genotype). On the other hand, phenotypes of both FC1 and FC2 were the results of synergetic interactions between indigenous rhizobia (most likely including parasitic rhizobia), inoculants, genotype and other environmental factors. However, the BNF capacity of small nodules (diameter $<2 \mathrm{~mm}$ ) was significantly lower than that of big nodules (diameter $>2 \mathrm{~mm}$ ) (Li et al., 2018). Contrastingly, FC1 seemed to have a higher compatibility with the inoculated rhizobial strains than indigenous soil rhizobia as indicated by great increases of NN and NW after rhizobium inoculation, especially at low N level (Figure 1). Since highly effective and affinitive rhizobial species are necessary for high BNF capacities to produce high yields in soybean (Peoples et al., 2009; Qin et al., 2012; Yang et al., 2017), TNC was measured for the RIL population to evaluate the effect of a highly effective and affinitive rhizobial species on soybean BNF capacity in the field. As expected, inoculation with highly effective and affinitive rhizobia led to $44.5 \%$ and $25.1 \%$ increase of TNC of RILs in low and high $\mathrm{N}$ conditions, respectively (Figure 2). Furthermore, the mean value of TNC in RILs did not significantly vary between $\mathrm{HN}-\mathrm{R}$ and $\mathrm{LN}+\mathrm{R}$ treatments, suggesting that rhizobium inoculation is an alternative way to meet $\mathrm{N}$ demand for soybean growth.

Nodulation traits that heavily influence BNF capacity are affected by many environment factors, such as nutrient availability, soil characteristics, abiotic stress, and soil tillage systems (Soedarjo et al., 2003; Adak and Kibritci, 2016; Chetan et al., 2016; Kunert et al., 2016). In soybean, although symbiotic $\mathrm{N}_{2}$ fixation can provide the nitrogen needed for plant growth, symbiotically fixed $\mathrm{N}$ is not always sufficient for producing high yields (Kunert et al., 2016). High $\mathrm{N}$ fertilizer application on the other hand has a negative impact on nodulation 

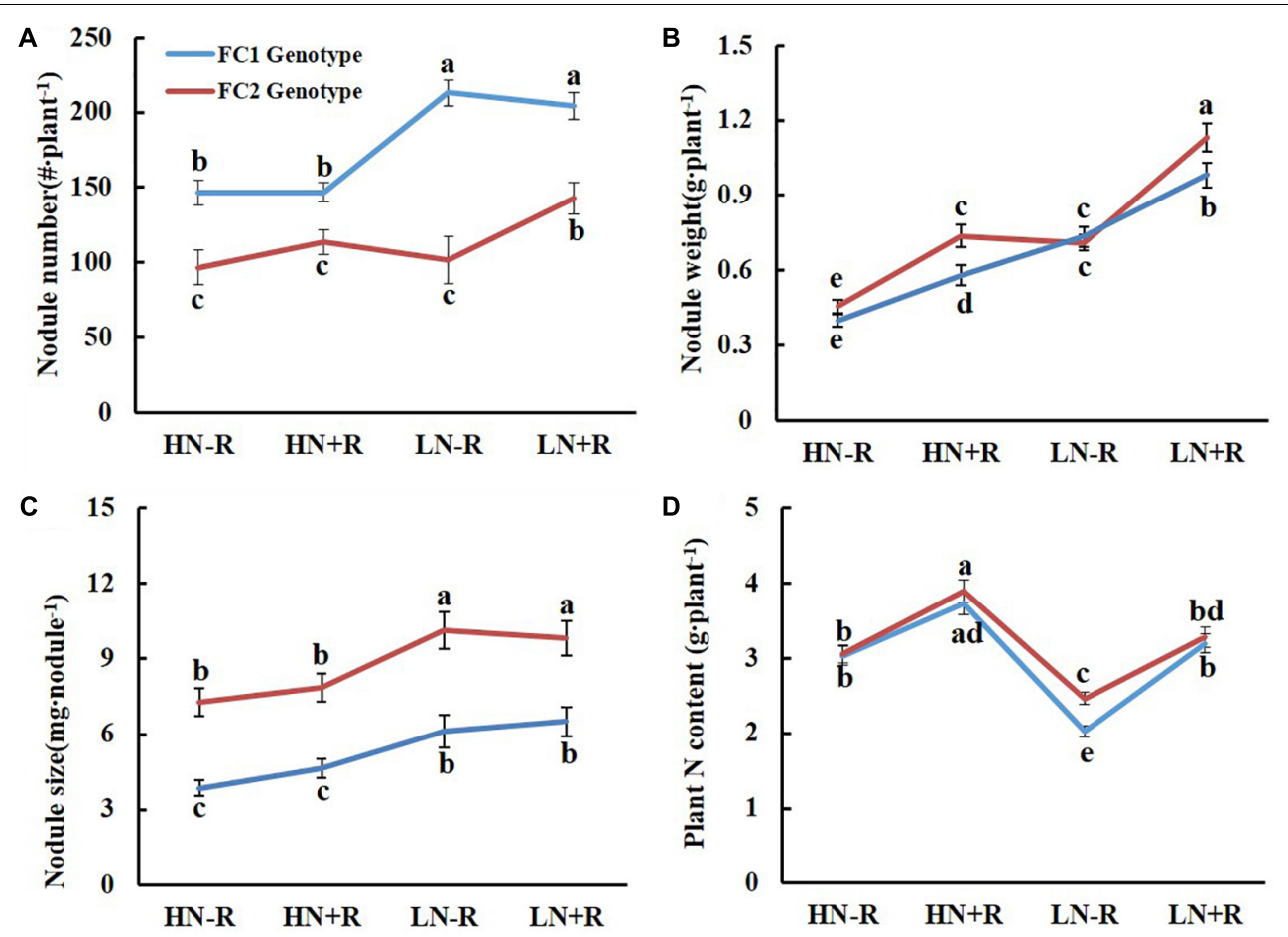

FIGURE 4 | Effect of qBNF-17 genotypes on nodulation traits. (A) Nodule number; (B) nodule weight; (C) nodule size; and (D) whole plant nitrogen content. Based on SNP marker Chr17.12638388, the RIL population was divided into FC1 and FC2 genotype groups which consisted of 83 and 81 lines, respectively. The blue and red line represented mean \pm SE values for the FC1 and FC2 genotype groups. Different letter(s) over the standard error bar indicated significant differences of mean values at the $5 \%$ level.

(Ferguson et al., 2010). In this study, BNF traits was studied in a field with low $\mathrm{N}$ and high $\mathrm{N}$ plots in which variation in nodule formation could proceed with or without rhizobial inoculation. As expected, the mean values of three BNF traits (i.e., NN, NW and NS) were significantly higher by $32.2-62.3 \%$ in low $\mathrm{N}$ plots than in high $\mathrm{N}$ plots, indicating that BNF capacities are higher when $\mathrm{N}$ is limited (Table 3). This is consistent with previous reports that high $\mathrm{N}$ status has a negative impact on nodulation traits (Ferguson et al., 2010; Abera and Tadele, 2016; Adak and Kibritci, 2016; Hu et al., 2016; Xia et al., 2017).

Genetics are the internal elements that might affect soybean BNF capacity along with environmental elements. Therefore, identification and mapping of QTLs for BNF traits holds promise for breeding efforts seeking to develop soybean varieties with high BNF capacities, which will allow growers to reduce $\mathrm{N}$ fertilization and maintain beneficial eco-systems. To successfully reach these goals, a high $h_{b}^{2}$ is necessary for identified solid QTLs. In this study, the $h^{2}{ }_{b}$ for NW and NS are stable under different environments, with values ranging from 0.62 to 0.70 and 0.70 to 0.87 , respectively. For $\mathrm{NN}$, rhizobial inoculation significantly decreased values of $h^{2}{ }_{b}$ to 0.48 and 0.63 in $\mathrm{HN}$ and LN field plots (Table 3), respectively, while other $h^{2}{ }_{b}$ values for nodulation traits remain above 0.75 without rhizobial inoculation under both $\mathrm{LN}$ and $\mathrm{HN}$ field conditions. This high level of heritability is consistent with previous reports (Souza et al., 2000; Santos et al., 2013; Yang et al., 2017). This strongly indicates that genetics is the main force affecting nodulation trait variation in the field, and, furthermore, the RIL population developed in this work is suitable for QTL identification.

Nodulation traits are difficult to quantify and study in field conditions. Although more than 20 QTLs for BNF traits have been identified in previous studies ${ }^{1}$, most of them were not adequately tested in field conditions, or there is a lack of adequate repetition required for obtaining precise QTL effect estimates. In addition, a high resolution genetic map is also necessary for precise QTL identification. Unfortunately, none of previous QTLs reports built on SSR based genetic maps exhibit adequate precision, possibly due to technical limitations. Therefore, it is not surprising that among previously identified QTLs for nodulation traits, relatively low LOD values ranging from 1.28 to 8.77 were detected, and none of them could explain more than $40 \%$ of variation (López-Bucio et al., 2003; Tanya et al., 2005; Nicolás et al., 2006; Santos et al., 2013; Yang et al., 2017). In this study, QTLs were precisely identified by using six representative plants for phenotypic observations, while a high resolution genetic map was constructed to detect QTLs under multiple environments. As a result of this effort, a QTL, $q B N F-$ 16 , explaining as much as $59 \%$ of genetic variation, and with LOD values of up to 32.52 was identified and mapped. Most importantly, $q B N F-16$ is a stable QTL that was detected under all four tested environments. Therefore, $q B N F-16$ can be considered

${ }^{1}$ https://www.soybase.org/ 
as a valuable locus which might be useful for breeding high BNF capacity soybean varieties.

Integration of QTLs for BNF traits in a previous reported reveals that a total of 18 QTLs affect BNF traits under field conditions (see footnote 1). These QTLs are distributed on linkage groups D1a(2), C1(1), A1(1), C2(4), A2(1), K(1), B1(1), $\mathrm{E}(2), \mathrm{L}(2)$, and I(3). None of these QTLs co-locate or are closely linked with QTLs identified in our study, suggesting that $q B N F$ 16 and $q B N F-17$ are two new QTLs. Taken together, in this study, two new QTLs for soybean nodulation and BNF capacity traits in the field conditions were identified and tested under different environments. The results indicate that these QTLs can be used as molecular markers for breeding elite soybean varieties with high BNF capacities.

\section{DATA AVAILABILITY STATEMENT}

Any related SNPs data can be available on request by contacting the corresponding author directly.

\section{AUTHOR CONTRIBUTIONS}

HLi designed the experiments and critically revised the manuscript. QY and YY analyzed the data and wrote the manuscript. QY, YY, RX, and HLv carried out the experiments.

\section{REFERENCES}

Abera, H., and Tadele, B. (2016). Effect of Rhizobium inoculation and nitrogen fertilization on nodulation and yield response of common bean (Phaseolus vulgaries L.) at Boloso Sore, Southern Ethiopia. Int. Jagric Biol. 6, 2224-2225.

Adak, M. S., and Kibritci, M. (2016). Effect of nitrogen and phosphorus levels on nodulationand yield components in faba bean (Vicia faba L.). Legume Res. 39, 991-994. doi: 10.18805/lr.v0iOF.3773

Adelson, P. A., Marcelo, G. T., and Dejair, L. D. A. (2000). Growth and yield of common bean cultivars at two soil phosphorus levels under biological nitrogen fixation. Pesqui Agropecu Bras. 35, 809-817. doi: 10.1590/S0100204X2000000400019

Aziz, A., Ahiabor, B., Opoku, A., and Abaidoo, R. (2016). Contributions of Rhizobium inoculants and phosphorus fertilizer to biological nitrogen fixation, growth and grain yield of three soybean varieties on a Fluvic Luvisol. Exp. Agric. 10, 1-11. doi: 10.9734/ajea/2016/20072

Bao, S. D. (2000). Soil and Agricultural Chemistry Analysis. Beijing: China Agricultural Press.

Chang, J. H., and Lee, W. S. (2004). A sliding window method for finding recently frequent itemsets over online data streams. J. Inf. Sci. Eng. 20, 753-762.

Chen, L. Y., and Liao, H. (2017). Engineering crop nutrient efficiency for sustainable agriculture. J. Integr. Plant Biol. 59, 710-735. doi: 10.1111/jipb. 12559

Cheng, F., Cao, G., Wang, X., Zhao, J., Yan, X., and Liao, H. (2009). Isolation and application of effective nitrogen fixation rhizobial strains on low-phosphorus acid soils in South China. Sci. Bull. 54, 412-420. doi: 10.1007/s11434-0080521-0

Chetan, C., Rusu, T., Chetan, F., and Simon, A. (2016). Influence of soil tillage systems and weed control treatments on root nodules, production and qualitative indicators of soybean. Procedia Technol. 22, 457-464. doi: 10.1016/j. protcy.2016.01.088

Colin, D. G., and Paul, R. K. (2012). IBM SPSS statistics 19 made simple. J. R. Stat. Soc. 4:1183.

\section{FUNDING}

This work was jointly supported by China National Key Program for Research and Development (2016YFD0100700), the Strategic Priority Research Program of the Chinese Academy of Sciences (XDB15030202), and National Natural Science Foundation of China (31601814).

\section{ACKNOWLEDGMENTS}

We would like to acknowledge staff members of the Institute of Genetics and Developmental Biology, Chinese Academy of Sciences, especially Yiping Tong for providing HN and LN level experimental farm sites, and Weiqi Zhu for field management, the graduate students of the Root Biology Center, Fujian Agriculture and Forestry University for soybean harvesting, and Dr. Thomas Walk of Golden Fidelity LLC for critical reviewing.

\section{SUPPLEMENTARY MATERIAL}

The Supplementary Material for this article can be found online at: https://www.frontiersin.org/articles/10.3389/fpls.2019.00075/ full\#supplementary-material

Denison, R. F., and Kiers, E. T. (2004). Lifestyle alternatives for rhizobia: mutualism, parasitism, and forgoing symbiosis. FEMS Microbiol. Lett. 237, 187-193. doi: 10.1016/j.femsle.2004.07.013

Elshire, R. J., Glaubitz, J. C., Sun, Q., Poland, J. A., Kawamoto, K., Buckler, E. S., et al. (2011). A robust, simple genotyping-by-sequencing (GBS) approach for high diversity species. PLoS One 6:e19379. doi: 10.1371/journal.pone.0019379

Ferguson, B. J., Indrasumunar, A., Hayashi, S., Lin, M. H., Lin, Y. H., Reid, D. E., et al. (2010). Molecular analysis of legume nodule development and autoregulation. J. Integr. Plant Biol. 52, 61-76. doi: 10.1111/j.1744-7909.2010. 00899.x

Fox, A. R., Soto, G., Valverde, C., Russo, D., Lagares, A., Zorreguieta, A., et al. (2016). Major cereal crops benefit from biological nitrogen fixation when inoculated with the nitrogen-fixing bacterium Pseudomonas protegens Pf-5 X940. Environ. Microbiol. 18, 3522-3534. doi: 10.1111/1462-2920.13376

Herridge, D. F., Peoples, M. B., and Boddey, R. M. (2008). Global inputs of biological nitrogen fixation in agricultural systems. Plant Soil 311, 1-18. doi: 10.1007/s11104-008-9668-3

Hu, F., Zhao, C., Feng, F., Chai, Q., Mu, Y., and Zhang, Y. (2016). Improving N management through intercropping alleviates the inhibitory effect of mineral $\mathrm{N}$ on nodulation in pea. Plant Soil 412, 235-251. doi: 10.1007/s11104-01630632

Jemo, M., Sulieman, S., Bekkaoui, F., Olomide, O. A. K., Hashem, A., Abd Allah, E. F., et al. (2017). Comparative analysis of the combined effects of different water and phosphate levels on growth and biological nitrogen fixation of nine cowpea varieties. Front. Plant Sci. 8:2111. doi: 10.3389/fpls.2017.02111

Joseph, M. J., Soon, G. L., and Ashley, M. S. (2016). The next green movement: plant biology for the environment and sustainability. Science 353, 1241-1244. doi: $10.1126 /$ science.aag 1698

Kumudini, S., Omielan, J., and Hume, D. J. (2008). Soybean genetic improvement in yield and the effect of late-season shading and nitrogen source and supply. Agron. J. 100, 278-290. doi: 10.2134/agronj2006.0201

Kunert, K. J., Vorster, B. J., Fenta, B. A., Kibido, T., Dionisio, G., and Foyer, C. H. (2016). Drought stress responses in soybean roots and nodules. Front. Plant Sci. 7:1015. doi: 10.3389/fpls.2016.01015 
Li, H., and Durbin, R. (2009). Fast and accurate short read alignment with Burrows-Wheeler transform. Bioinformatics 25, 1754-1760. doi: 10.1093/ bioinformatics/btp324

Li, X., Zeng, R., and Liao, H. (2016). Improving crop nutrient efficiency through root architecture modifications. J. Integr. Plant Biol. 58, 193-202. doi: 10.1111/ jipb.12434

$\mathrm{Li}, \mathrm{X}$., Zheng, J., Yang, Y., and Liao, H. (2018). Increasing nodule size1 expression is required for normal rhizobial symbiosis and nodule development. Plant Physiol. 178, 1233-1248. doi: 10.1104/pp.18.01018

López-Bucio, J., Cruz-Ramìrez, A., and Herrera-Estrella, L. (2003). The role of nutrient availability in regulating root architecture. Curr. Opin. Plant Biol. 6, 280-287. doi: 10.1016/s1369-5266(03)000359

Meng, L., Li, H., Zhang, L., and Wang, J. (2015). QTL IciMapping: integrated software for genetic linkage map construction and quantitative trait locus mapping in biparental populations. Crop J. 3, 269-283. doi: 10.1016/j.cj.2015. 01.001

Menna, P., Hungria, M., Barcellos, F. G., Bangel, E. V., Hess, P. N., and MartinezRomero, E. (2006). Molecular phylogeny based on the 16S rRNA gene of elite rhizobial strains used in Brazilian commercial inoculants. Syst. Appl. Microbiol. 29, 315-332. doi: 10.1016/j.syapm.2005.12.002

Nascimento, V. C. D., Prete, C. E. C., and Nogueira, M. A. (2016). Biological nitrogen fixation in soybean under water restriction and exposed to 1methylcyclopropene. Pesq. Agropec. Bras. 51, 818-823. doi: 10.1590/s0100$204 \times 2016000700004$

Nicolás, M. F., Hungria, M., and Arias, C. A. A. (2006). Identification of quantitative trait loci controlling nodulation and shoot mass in progenies from two Brazilian soybean cultivars. Field Crop Res. 95, 355-366. doi: 10.1016/j.fcr. 2005.04.012

Nohara, T., Nakayama, N., Takahashi, M., Maruyama, S., Shimada, S., and Arihara, J. (2005). Cultivar differences in dependence on nitrogen fixation of soybeans in the field with a high soil nitrate level determinated by the relative ureide abundance method. Proc. Crop Sci. Soc. Jpn. 74, 316-324. doi: 10.1626/ jcs.74.316

Peoples, M. B., Brockwell, J., Herridge, D. F., Rochester, I. J., Alves, B. J. R., and Urquiaga, S. (2009). The contributions of nitrogen-fixing crop legumes to the productivity of agricultural systems. Symbiosis 48, 1-17. doi: $10.1007 /$ BF03179980

Qin, L., Zhao, J., Tian, J., Chen, L., Sun, Z., Guo, Y., et al. (2012). The highaffinity phosphate transporter GmPT5 regulates phosphate transport to nodules and nodulation in soybean. Plant Physiol. 159, 1634-1643. doi: 10.1104/pp.112. 199786

Santos, M. A., Geraldi, I. O., Garcia, A. A. F., Bortolatto, N., Schiavon, A., and Hungria, M. (2013). Mapping of QTLs associated with biological nitrogen fixation traits in soybean. Hereditas 150, 17-25. doi: 10.1111/j.1601-5223.2013. 02275.x

Sivparsad, B. J., Chiuraise, N., and Laing, M. D. (2015). Comparative evaluation of commercial rhizobial inoculants of soybean. South Afr. J. Plant Soil 33, 157-160. doi: 10.1080/02571862.2015.1092177

Soedarjo, M., Saleh, N., Adisarwanto, T., and Ishiki, K. (2003). Characterization and effectiveness of rhizobia isolated from nodules of soybean grown on al-rich acid soils. Jpn. J. Trop. Agric. 47, 63-64.

Souza, A. A., Boscariol, R. L., Moon, D. H., Camargo, L. E. A., and Tsai, S. M. (2000). Effects of Phaseolus vulgaris QTL in controlling host-bacteria interactions under two levels of nitrogen fertilization. Genet. Mol. Biol. 23, 155-161. doi: 10.1590/S1415-47572000000100029
Takahashi, R., Dubouzet, J. G., Matsumura, H., Yasuda, K., and Iwashina, T. (2010). A new allele of flower color gene $W 1$ encoding flavonoid $3^{\prime} 5^{\prime}$-hydroxylase is responsible for light purple flowers in wild soybean Glycine soja. BMC Plant Biol. 10:155. doi: 10.1186/1471-2229-10-155

Tanya, P., Srinives, P., Toojinda, T., Vanavichit, A., and Lee, S. H. (2005). Identification of SSR markers associated with N2-fixation components in soybean [Glycine $\max$ (L.) Merr.]. Korean J. Genet. 27, 351-359.

Thomas-Oates, J., Bereszczak, J., Edwards, E., Gill, A., Noreen, S., Zhou, J. C., et al. (2003). A catalogue of molecular, physiological and symbiotic properties of soybean-nodulating rhizobial strains from different soybean cropping areas of China. Syst. Appl. Microbiol. 26, 453-465. doi: 10.1078/0723202033224 97491

Thuita, M., Pypers, P., Herrmann, L., Okalebo, R. J., Othieno, C., Muema, E., et al. (2012). Commercial rhizobial inoculants significantly enhance growth and nitrogen fixation of a promiscuous soybean variety in Kenyan soils. Biol. Fertil. Soils 48, 87-96. doi: 10.1007/s00374-011-0611-z

Udvardi, M., and Poole, P. S. (2013). Transport and metabolism in legume-rhizobia symbioses. Annu. Rev. Plant Biol. 64, 781-805. doi: 10.1146/annurev-arplant050312-120235

Van Ooijen, J. W. (2009). MapQTL 6, Software for the Mapping of Quantitative Trait Loci in Experimental Populations of Diploid Species. Wageningen: Kyazma B.V.

Wang, K., Li, M., and Hakonarson, H. (2010). ANNOVAR: functional annotation of genetic variants from high-throughput sequencing data. Nucleic Acids Res. 38:e164. doi: 10.1093/nar/gkq603

Ward, A. (2011). Phosphorus Limitation of Soybean and Alfalfa Biological Nitrogen Fixation on Organic Dairy Farms. M.Sc. thesis, NSAC, Bible Hill.

Xia, X., Ma, C., Dong, S., Xu, Y., and Gong, Z. (2017). Effects of nitrogen concentrations on nodulation and nitrogenase activity in dual root systems of soybean plants. J. Soil Sci. Plant Nutr. 63, 1-13. doi: 10.1080/00380768.2017. 1370960

Yang, Y., Zhao, Q., Li, X., Ai, W., Liu, D., Qi, W., et al. (2017). Characterization of genetic basis on synergistic interactions between root architecture and biological nitrogen fixation in soybean. Front. Plant Sci. 8:1466. doi: 10.3389/ fpls.2017.01466

Yang, Z. W., Shen, Y. Y., Xie, T. L., and Tan, G. Y. (2009). Biological nitrogen fixation efficiency in soybean under different levels of nitrogen supply. Acta Bot. Boreali Occident Sin. 29, 574-579.

Yendrek, C. R., Lee, Y.-C., Morris, V., Liang, Y., Pislariu, C. I., Burkart, G., et al. (2010). A putative transporter is essential for integrating nutrient and hormone signaling with lateral root growth and nodule development in Medicago truncatula. Plant J. 62, 100-112. doi: 10.1111/j.1365-313X.2010. 04134.x

Conflict of Interest Statement: The authors declare that the research was conducted in the absence of any commercial or financial relationships that could be construed as a potential conflict of interest.

Copyright (c) 2019 Yang, Yang, Xu, Lv and Liao. This is an open-access article distributed under the terms of the Creative Commons Attribution License (CC BY). The use, distribution or reproduction in other forums is permitted, provided the original author(s) and the copyright owner(s) are credited and that the original publication in this journal is cited, in accordance with accepted academic practice. No use, distribution or reproduction is permitted which does not comply with these terms. 